\title{
INDECOMPOSABLE CONTINUA AND THE JULIA SETS OF POLYNOMIALS
}

\author{
JOHN C. MAYER AND JAMES T. ROGERS, JR.
}

(Communicated by James E. West)

\begin{abstract}
We find several necessary and sufficient conditions for the Julia set $J$ of a polynomial of degree $d \geq 2$ to be an indecomposable continuum. One condition that may be easier to check than others is the following: Suppose $J$ is connected; then $J$ is an indecomposable continuum iff the impression of some prime end of the unbounded complementary domain of $J$ has interior in $J$.
\end{abstract}

\section{INTRODUCTION}

The Julia set $J(f)$ of a polynomial $f$ of degree $d \geq 2$ is always a compact, perfect, nonempty subset of the complex plane $\mathbf{C}$. When the critical points of $f$ all have bounded orbits, $J(f)$ is a continuum [M, Theorem 17.3]. Beginning with classical work of Julia and Fatou, and more recently through the work of Branner, Douady, Herman, Hubbard, Lyubich, Yoccoz, and others, an understanding of the topological structure of the Julia sets of polynomials is growing, at least in those cases where the Julia set is locally connected. An excellent introduction to the current state of affairs is Milnor's lecture notes [M].

As Sullivan and Douady have observed, however, there are polynomials with non-locally-connected Julia sets, even in the family of quadratic polynomials [M, Corollary 18.6]. In fact, Douady has shown in an unpublished proof that there are Julia sets in the quadratic family whose unbounded complementary domain contains a prime end of the third (or fourth) kind. The topological structure of these non-locally-connected Julia sets remains a mystery. In particular, how badly non-locally-connected they may be is unknown. It is that mystery which motivates our study herein of necessary and sufficient conditions for the Julia set of a polynomial to be an indecomposable continuum. Our main results are Theorems 3.2 and 3.4. We also raise some questions (2.3, 3.6-3.8). Additional questions about non-locally-connected Julia sets may be found in [B].

Received by the editors July 2, 1991; portions of this paper were presented by the first author at the Spring Topology Conference, Sacramento, CA, April 11-13, 1991, and by the second author at the Conference and Workshop on Continuum Theory and Dynamical Systems, Lafayette, LA, May $17-20,1991$.

1991 Mathematics Subject Classification. Primary 30C35, 54F20.

Key words and phrases. Julia set, indecomposable continuum, internal composant, prime end, simple dense canal, Lake of Wada, complex analytic dynamics, conformal dynamics. 
1.1. Prime end uniformization. Let $U \subset \widehat{\mathbf{C}}$ be a simply connected domain with nondegenerate boundary and $\phi: \mathbf{D} \rightarrow U$ a complex analytic homeomorphism, as guaranteed by the Riemann Mapping Theorem. We call $\phi$ a (prime end) uniformization of $U$. Each prime end of $U$ corresponds to a unique point $\eta \in$ $\partial D$. Prime end theory was introduced by Carathéodory to study the approaches to the boundary of a simply connected domain. The facts about prime end theory summarized below may be found in [P, CL, M, Br]. For convenience, we identify $\mathbf{R} / \mathbf{Z}=\partial \mathbf{D}$, and by $\eta$ we denote both the point $\eta \in \partial \mathbf{D}$ and the prime end $\eta$ of $U$ corresponding to that point.

Suppose $\phi: \mathbf{D} \rightarrow U$ is a uniformization and $\eta \in \partial \mathbf{D}=\mathbf{R} / \mathbf{Z}$. Let $R_{\eta}=$ $\phi([0, \eta))$ denote the image in $U$ of the radial ray

$$
[0, \eta)=\left\{z \in \mathbf{C} \mid z=r e^{2 \pi i \eta} \text { and } 0 \leq r<1\right\}
$$

in D. The radial limit set (also called principal set) at $\eta$ is the continuum $P(\eta)=\overline{R_{\eta}}-R_{\eta}$ in $\partial U$. The impression of the prime end $\eta$ is the continuum

$$
I(\eta)=\left\{w \in \widehat{\mathbf{C}} \mid \exists\left\{z_{i}\right\} \subset \mathbf{D}, z_{i} \underset{i}{\rightarrow} \eta \text { and } \phi\left(z_{i}\right) \underset{i}{\rightarrow} w\right\}
$$

contained in $\partial U$. There exists a ray (i.e., half-open arc) $S_{\eta}$ in $\mathbf{D}$ from 0 to $\eta$, not necessarily radial, such that $I(\eta)=\overline{\phi\left(S_{\eta}\right)}-\phi\left(S_{\eta}\right)$. When the radial limit set is a point $p \in \partial U$, we say $R_{\eta}$ lands on $p$. A point $p \in \partial U$ is accessible from $U$ iff there exists a ray $R$ in $U$ such that $\bar{R}-R=\{p\}$. A point $p \in \partial U$ is accessible with respect to a prime end $\eta$ of $U$ iff $P(\eta)=\{p\}$. Each point of $\partial U$ accessible from $U$ is accessible with respect to at least one prime end of $U$.

Closer to Carathéodory's original formulation is the definition of prime ends in terms of chains of crosscuts of $U$. In this connection, we have

$$
\begin{gathered}
P(\eta)=\left\{w \in \widehat{\mathbf{C}} \mid \exists \text { a chain }\left\{Q_{i}\right\}_{i=1}^{\infty}\right. \text { of crosscuts } \\
\text { in } \left.U \text { defining } \eta \text { with } Q_{i} \underset{i}{\rightarrow} w\right\}, \\
I(\eta)=\bigcap_{i=1}^{\infty} \overline{U_{i}}=\bigcap_{i=1}^{\infty} \partial U_{i},
\end{gathered}
$$

where $U_{i}$ is the component of $U-Q_{i}$ which contains $Q_{j}$ for all $j>i$ in the chain $\left\{Q_{i}\right\}_{i=1}^{\infty}$ of crosscuts defining a prime end $\eta$.

\subsection{Kinds of prime ends. Consider the conditions}

(1) $P(\eta)$ is degenerate.

(2) $I(\eta)=P(\eta)$.

A prime end $\eta$ is of the first, second, third, or fourth kind accordingly, as $\eta$ satisfies both (1) and (2), (1) only, (2) only, or neither (1) nor (2). For a given domain $U$, let $E_{i}=E_{i}(U)$ denote the set of points in $\partial \mathbf{D}$ corresponding to prime ends of the $i$ th kind. A simple dense canal (also called a Lake-of-Wada channel) is a prime end $\eta$ such that $I(\eta)=P(\eta)=\partial U$. In what follows, we will need the following theorem of Collingwood about kinds of prime ends $[P$, p. 50]: 


\subsection{Theorem. $E_{1} \cup E_{3}$ is a residual set in $\partial \mathbf{D}$.}

1.4. Polynomials. Suppose that $f: \widehat{\mathbf{C}} \rightarrow \widehat{\mathbf{C}}$ is a polynomial of degree $d \geq 2$. By $J(f)$ we denote the Julia set (unstable set), by $F(f)$ the Fatou set (stable set), and by $K(f)$ the filled-in Julia set of $f$ (that is, $K(f)=$ the union of $J(f)$ and its bounded complementary domains, if any). (See [M] or [DH1] for definitions.)

We will limit ourselves to polynomials for which $J(f)$ is connected. In this case, $J(f)=\partial K(f)=\partial(\widehat{\mathbf{C}}-K(f))[\mathrm{M}$, Lemma 17.1]. We will be concerned with the prime end structure of the simply connected domain $U=\widehat{\mathbf{C}}-K(f)$, which is the domain of attraction of $\infty$, and the relationship between the prime end structure of $U$ and the topological structure of the boundary $\partial U=J(f)$. It will be convenient for us to use $\widehat{\mathbf{C}}-\overline{\mathbf{D}}$ as the uniformizing domain. There exists a Bötkher uniformization $\phi: \widehat{\mathbf{C}}-\overline{\mathbf{D}} \rightarrow U$ such that $\phi(\infty)=\infty$ and $\phi$ conjugates $f$ on $U$ to $h(z)=z^{d}$ on $\widehat{\mathbf{C}}-\overline{\mathbf{D}}$; that is, $f \circ \phi=\phi \circ h$ [M, Theorem 17.3]. For $\eta \in \partial \mathbf{D}$, Douady and Hubbard call the ray $R_{\eta}=\phi([\infty, \eta))$ in $U$ the external ray at $\eta$. Note that the map $z \rightarrow z^{d}$ on $\partial \mathbf{D}$ is $\eta \rightarrow d \eta$ on $\mathbf{R} / \mathbf{Z}$.

\section{Prime end lemmas}

We suppose throughout the remaining sections that $U$ is a simply connected domain in $\widehat{\mathbf{C}}$ with nondegenerate boundary. When so stated, we specialize to $U=\widehat{\mathbf{C}}-K(f)$ for $f$ a polynomial of degree $d \geq 2$ and $J(f)$ connected.

2.1. Lemma. Suppose that $\phi: \widehat{\mathbf{C}}-\overline{\mathbf{D}} \rightarrow U$ is any uniformization, $h(z)=z^{d}$ on $\widehat{\mathbf{C}}-\overline{\mathbf{D}}(d \geq 2)$, and $\phi \circ h \circ \phi^{-1}: U \rightarrow U$ extends continuously to $H: \bar{U} \rightarrow \bar{U}$. Then for all $\eta \in \partial \mathbf{D}, H(I(\eta))=I(d \eta)$ and $H(P(\eta))=P(d \eta)$.

Proof. Let $\eta \in \partial \mathbf{D}$. Let $x \in H(I(\eta))$. Choose $x_{0} \in I(\eta)$ such that $H\left(x_{0}\right)=x$. Then there exists $z_{i} \in \widehat{\mathbf{C}}-\overline{\mathbf{D}}$ such that $z_{i} \rightarrow \eta$ and $\phi\left(z_{i}\right) \rightarrow x_{0}$. Hence, $h\left(z_{i}\right) \rightarrow d \eta$ and $\phi \circ h\left(z_{i}\right)=H \circ \phi\left(z_{i}\right) \rightarrow H\left(x_{0}\right)=x$. Thus, $x \in I(d \eta)$. Therefore, $H(I(\eta)) \subset I(d \eta)$.

Conversely, let $x \in I(d \eta)$. Then there exist $z_{i} \in \widehat{\mathbf{C}}-\overline{\mathbf{D}}$ such that $z_{i} \rightarrow d \eta$ and $\phi\left(z_{i}\right) \rightarrow x$. Since $h(z)=z^{d}$, we can pull back to find a sequence $z_{i}^{\prime} \epsilon$ $\widehat{\mathbf{C}}-\overline{\mathbf{D}}$, such that $h\left(z_{i}^{\prime}\right)=z_{i}$, and $z_{i}^{\prime} \rightarrow \eta$. Taking a subsequence if necessary, we may suppose $\phi\left(z_{i}^{\prime}\right) \rightarrow x^{\prime}$ for some $x^{\prime} \in \partial U$. Then $x^{\prime} \in I(\eta)$. Now $H \circ \phi\left(z_{i}^{\prime}\right)=\phi \circ h\left(z_{i}^{\prime}\right)=\phi\left(z_{i}\right) \rightarrow x$. By continuity of $H, H \circ \phi\left(z_{i}^{\prime}\right) \rightarrow H\left(x^{\prime}\right)$. Hence, we have $H\left(x^{\prime}\right)=x$. Thus, $x \in H(I(\eta))$. Therefore, $I(d \eta) \subset H(I(\eta))$.

Now consider $R_{\eta}=\phi([\infty, \eta)) \subset U$. Since $P(\eta)=\overline{R_{\eta}}-R_{\eta}$, we have $H(P(\eta))=H\left(\overline{R_{\eta}}-R_{\eta}\right)=H\left(\overline{R_{\eta}}\right)-H\left(R_{\eta}\right)$. Then we have $H\left(R_{\eta}\right)=H$ 。 $\phi([\infty, \eta))=\phi \circ h([\infty, \eta))=\phi([\infty, d \eta))=R_{d \eta}$. Also, $H\left(\overline{R_{\eta}}\right) \subset \overline{H\left(R_{\eta}\right)}$, by continuity of $H$. Hence,

$$
H(P(\eta))=H\left(\overline{R_{\eta}}\right)-H\left(R_{\eta}\right) \subset \overline{H\left(R_{\eta}\right)}-H\left(R_{\eta}\right)=\overline{R_{d \eta}}-R_{d \eta}=P(d \eta) .
$$

Therefore, $H(P(\eta)) \subset P(d \eta)$.

Conversely, let $x \in P(d \eta)$. Then $x \in \overline{R_{d \eta}}-R_{d \eta}$. Let $w_{i} \in R_{d \eta}$ such that $w_{i} \rightarrow x$. We can pull back to find a sequence $w_{i}^{\prime} \in R_{\eta}$ such that $H\left(w_{i}^{\prime}\right)=w_{i}$ and $w_{i}^{\prime} \rightarrow x^{\prime}$ for some $x^{\prime} \in \partial U$. Then $x^{\prime} \in P(\eta)$. Since $H\left(w_{i}^{\prime}\right)=w_{i} \rightarrow x$, we have $H\left(x^{\prime}\right)=x$. Thus, $x \in H(P(\eta))$. Therefore, $P(d \eta) \subset H(P(\eta))$. 
2.2. Lemma. Suppose that $f$ is a polynomial of degree $d \geq 2$ with $J=J(f)$ connected. Let $U=\widehat{\mathbf{C}}-K(f)$ and suppose $\phi: \widehat{\mathbf{C}}-\overline{\mathbf{D}} \rightarrow U$ is a uniformization such that $\phi(\infty)=\infty$ and $\phi$ conjugates $f$ to $h(z)=z^{d}$ on $\widehat{\mathbf{C}}-\overline{\mathbf{D}}$. If $\eta \in \partial \mathbf{D}$ such that $I(\eta)=J$, then $J=\bigcup_{\eta^{\prime} \in h^{-1}(\eta)} I\left(\eta^{\prime}\right)$.

Proof. Let $x \in J-\{$ critical points of $f\}$ and let $x_{0}=f(x)$. Since $I(\eta)=J$, there exists $\left\{z_{i}\right\} \subset \widehat{\mathbf{C}}-\overline{\mathbf{D}}$ such that $z_{i} \rightarrow \eta$ and $\phi\left(z_{i}\right) \rightarrow x_{0}$. Since $x_{0}$ is not a critical value, there are neighborhoods $N$ of $x$ and $f(N)$ of $x_{0}$ homeomorphic under $f$. We may assume $\phi\left(z_{i}\right) \in f(N)$, for all $i$. Since $U$ is fully invariant under $f$, there exist $q_{i} \in U \cap N$ such that $f\left(q_{i}\right)=\phi\left(z_{i}\right)$ and $q_{i} \rightarrow x$. Then

$$
\phi \circ h \circ \phi^{-1}\left(q_{i}\right)=\phi\left(z_{i}\right) \text {, }
$$

so $h \circ \phi^{-1}\left(q_{i}\right)=z_{i}$. As there are exactly $d$ preimages of $x_{0}$ in $J$, the $\phi^{-1}\left(q_{i}\right)$ 's converge to some one of the $d$ preimages $\eta^{\prime} \in h^{-1}(\eta)$. Hence, $x \in I\left(\eta^{\prime}\right)$. Since $J-\{$ critical points of $f\}$ is dense in $J$ and $I\left(\eta^{\prime}\right)$ is closed for each of the finitely many $\eta^{\prime} \in h^{-1}(\eta)$, it follows that $J \subset \bigcup_{\eta^{\prime} \in h^{-1}(\eta)} I\left(\eta^{\prime}\right)$.

Observe that the hypotheses of Lemma 2.2 require that $\phi \circ h \circ \phi^{-1}: U \rightarrow U$ extend analytically to $\bar{U}$, while the hypotheses of Lemma 2.1 require only a continuous extension. (Note that $f \mid \bar{U}$ in Lemma 2.2 is the extension called $H$ in Lemma 2.1.) This raises the following question:

2.3. Question. Does Lemma 2.2 hold under the weaker hypotheses of Lemma 2.1 ?

\section{INDECOMPOSABLE JULIA SETS}

Let $X$ be a nondegenerate indecomposable continuum contained in the complex plane $\mathbf{C}$. The union of all proper subcontinua containing a point $x$ of $X$ is called a composant of $X$. The collection of composants of $X$ forms a partition of $X$ into disjoint sets. The continuum $X$ contains uncountably many composants, and each composant is a dense $F_{\sigma}$ in $X$ [HY, p. 140].

A composant $C$ of $X$ is said to be internal iff every continuum $L$ intersecting both $C$ and the complement of $X$ intersects all composants of $X$. Otherwise $C$ is external. Krasinkiewicz introduced the notion of internal composants [K1] and proved that the union of the internal composants of $X$ is a nonempty $G_{\delta}$-subset of $X$ [K2]. It follows that the union of the external composants of $X$ is an $F_{\sigma}$-subset of $X$ (which, of course, is nonempty).

In this section, we shall use the following fact about internal composants [K2, Theorem 2.3]. (See also [Ru, Lemma 4; H, Theorem 1].)

3.1. Theorem. Let $X$ be a nonseparating plane continuum. If $Y$ is an indecomposable subcontinuum of the boundary of $X$ and $C$ is an internal composant of $Y$, then any subcontinuum of $X$ intersecting both $C$ and the complement of $Y$ contains $Y$.

The following theorem is the first of our main results. Perhaps condition (2) or (3) below will prove easier to verify (or refute) for a given Julia set $J$ than other well-known conditions equivalent to $J$ being indecomposable. 
3.2. Theorem. Suppose the continuum $J=J(f)$ is the Julia set of a polynomial $f$ of degree $d \geq 2$. Let $U=\widehat{\mathbf{C}}-K(f)$. The following are equivalent:

(1) $J$ is indecomposable.

(2) Some indecomposable subcontinuum of $J$ has nonempty interior in $J$.

(3) The impression of some prime end of $U$ has nonempty interior in $J$.

(4) The impression of some prime end of $U$ is equal to $J$.

Proof. (1) $\Rightarrow$ (2). Trivial.

(2) $\Rightarrow(3)$. This follows from [Ru, Theorem 4].

(3) $\Rightarrow(4)$. Let $V$ be an open set in $\mathbf{C}$ such that $V \cap J \subset I(\eta)$ for some prime end $\eta$ of $U$. For $n$ sufficiently large, the image $f^{n}(V \cap J)$ of $V \cap J$ is all of $J$ [M, Corollary 11.2]. Since $V \cap J \subset I(\eta)$, it follows from Lemma 2.1 that

$$
J=f^{n}(V \cap J) \subset f^{n}(I(\eta))=I\left(d^{n} \eta\right) .
$$

Hence, (4) is satisfied.

(4) $\Rightarrow(1)$. Suppose $I(\eta)=J$ for some prime end $\eta$ of $U$. From [Ru, Theorem 2], we know that either $J$ is indecomposable or $J$ is the union $A \cup B$ of two of its proper indecomposable subcontinua $A$ and $B$. In the latter case, the interior of $A$ and the interior of $B$ are nonempty, and so for sufficiently large $n, f^{n}(A)=A \cup B=f^{n}(B)$, by [M, Corollary 11.2]. It follows that the image under $f^{n}$ of each composant of $A$ and of each composant of $B$ is dense in $A \cup B$.

Let

$$
\begin{aligned}
& \mathscr{A}=\left\{C \mid C \text { is a composant of } A \text { and either } f^{n}(C) \supset A \text { or } f^{n}(C) \supset B\right\}, \\
& \mathscr{B}=\left\{C \mid C \text { is a composant of } B \text { and either } f^{n}(C) \supset A \text { or } f^{n}(C) \supset B\right\} .
\end{aligned}
$$

Since $f^{n}$ is finite-to-one, both $\mathscr{A}$ and $\mathscr{B}$ contain only a finite number of composants. Hence, $\mathscr{A}^{*}$ and $\mathscr{B}^{*}$ (the union of the composants in $\mathscr{A}$ and $\mathscr{B}$, respectively) are each $F_{\sigma}$-subsets of $J$ with empty interior.

We claim that each composant $C$ of either $A$ or $B$ that does not belong to $\mathscr{A} \cup \mathscr{B}$ has the property that

$$
f^{n}(C) \subset \operatorname{Ext}(A) \cup \operatorname{Ext}(B),
$$

where $\operatorname{Ext}(A)$ denotes the union of the external composants of $A$. To see this, one must check four similar cases; we check one of them: suppose $C$ is a composant of $A$ not in $\mathscr{A}$. Since $f^{n}(C)$ is dense in $A \cup B$, there is a continuum $Z \subset f^{n}(C)$ which meets both $A-B$ and $B$. Suppose $Z$ meets a composant $D$ of $B$, and by way of contradiction, suppose $D$ is an internal composant of $B$. Then by Theorem $3.1, Z$ contains $B$, implying $f^{n}(C)$ contains $B$, a contradiction of $A \notin \mathscr{A}$.

Since $\operatorname{Ext}(A)$ and $\operatorname{Ext}(B)$ are $F_{\sigma}$ 's [K2, Theorem 3.2], so is $\operatorname{Ext}(A) \cup \operatorname{Ext}(B)$, and hence, $\left(f^{n}\right)^{-1}(\operatorname{Ext}(A) \cup \operatorname{Ext}(B))$ is an $F_{\sigma}$. Since

$$
A \cup B=\mathscr{A}^{*} \cup \mathscr{B}^{*} \cup\left(f^{n}\right)^{-1}(\operatorname{Ext}(A) \cup \operatorname{Ext}(B)),
$$

it follows from the Baire Category Theorem that $\left(f^{n}\right)^{-1}(\operatorname{Ext}(A) \cup \operatorname{Ext}(B))$ contains an open set $W$. Then $f^{n}(W) \subset \operatorname{Ext}(A) \cup \operatorname{Ext}(B)$, a set with empty interior in $J$. Since the Julia set $J$ is completely invariant, the restriction of $f^{n}$ to $J$ is an open map of $J$ onto itself. This contradiction proves the theorem.

The Baire Category Theorem implies the following corollary. 
3.3. Corollary. Suppose the continuum $J=J(f)$ is the Julia set of a polynomial $f$ of degree $d \geq 2$. Let $U=\widehat{\mathbf{C}}-K(f)$. If $J$ is either the union of a countable number of indecomposable continua or a countable number of impressions of prime ends of $U$, then $J$ is an indecomposable continuum.

The following theorem and corollary give us some insight into what an indecomposable Julia set $J$ of a quadratic polynomial would have to look like. The domain of attraction $U$ of infinity would have to have ubiquitous Lakeof-Wada channels, each dense in the continuum $J$ bounding $U$. Moreover, no composant of $J$ could contain more than one point accessible from $U$. In addition, no point $x$ of $J$ could be accessible with respect to more than one prime end of any complementary domain $V$ of $\widehat{\mathbf{C}}-J$; otherwise, one could construct a simple closed curve in $V \cup\{x\}$ that would show $x$ was a separating point of $J$, contradicting $J$ being indecomposable.

The pseudocircle in certain embeddings in $\mathbf{C}$ is an example of a continuum with the properties described above. This example, and simpler (not hereditarily indecomposable) examples, are discussed in [BGM, MO]. (Also see [R1].) These examples, however, all separate the plane into exactly two complementary domains.

Theorem 3.4 and its corollary ought to be true for any polynomial $f$ of degree $d \geq 2$; however, our proof only works for polynomials with symmetric Julia sets, that is, those invariant under multiplication by a $d$ th root of unity. We prove the theorem only for $d=2$.

3.4. Theorem. Suppose $f_{c}(z)=z^{2}+c$ with $J=J\left(f_{c}\right)$ connected. Let $U$ be the domain $\widehat{\mathbf{C}}-K\left(f_{c}\right)$. Suppose that $\phi: \widehat{\mathbf{C}}-\overline{\mathbf{D}} \rightarrow U$ is a uniformization such that $\phi(\infty)=\infty$ and $\phi$ conjugates $f_{c}$ on $U$ to $f_{0}$ on $\widehat{\mathbf{C}}-\overline{\mathbf{D}}$. Then the following are equivalent:

(1) The Julia set $J$ is indecomposable.

(2) For some prime end $\eta$ of $U, I(\eta)=J$.

(3) For a dense subset $\mathscr{D}$ of $\partial \mathbf{D}$, for each $\eta \in \mathscr{D}, I(\eta)=J$.

(4) For every prime end $\eta$ of $U, I(\eta)=J$.

(5) The set of simple dense canals of $U$ is a residual set in $\partial \mathbf{D}$.

(6) Some prime end $\eta$ of $U$ is a simple dense canal.

Proof. (1) $\Leftrightarrow(2)$. By Theorem 3.2, (1) and (2) are equivalent.

(2) $\Leftrightarrow(3)$. Suppose $\eta$ is a prime end of $U$ for which $I(\eta)=J$. Let $\eta_{1}$ and $\eta_{2}=\eta_{1}+1 / 2$ be the two preimages of $\eta$ in $\partial \mathbf{D}$ under $f_{0}$. Then by Lemma $2.2, J=I\left(\eta_{1}\right) \cup I\left(\eta_{2}\right)$. Now $K\left(f_{c}\right)$ is symmetric with respect to rotation by $\pi$ radians $\left(z \rightarrow-z=e^{\pi i} z\right)$. Since $\phi\left(z^{2}\right)=\phi\left(f_{0}(z)\right)=f_{c}(\phi(z))=\phi(z)^{2}+c$ and $\phi$ is one-to-one, a calculation shows that $\phi(-z)=-\phi(z)$. Hence, $I\left(\eta_{1}\right)$ is ambiently homeomorphic to $I\left(\eta_{2}\right)$. (To see this, imitate the proof of Lemma 2.1.) Thus, either both $I\left(\eta_{1}\right)$ and $I\left(\eta_{2}\right)$ are proper subcontinua of $J$ or neither is. Since $J$ is indecomposable (by $(2) \Rightarrow(1)$ ), we have $I\left(\eta_{1}\right)=J=I\left(\eta_{2}\right)$.

The set

$$
\mathscr{D}=\left\{\eta^{\prime} \in \partial \mathbf{D} \mid f_{0}^{n}\left(\eta^{\prime}\right)=2^{n} \eta^{\prime}=\eta \text { for some } n=0,1,2, \ldots\right\}
$$

of iterated preimages of $\eta$ under $f_{0}$ is dense in $\partial \mathbf{D}$. By applying the argument in the preceding paragraph inductively, $I\left(\eta^{\prime}\right)=J$ for all $\eta^{\prime} \in \mathscr{D}$. 
(3) $\Rightarrow(4)$. Suppose $\mathscr{D}$ is a dense set of points of $\partial \mathbf{D}$ corresponding to prime ends with impression all of $J$. Then for any crosscut $Q$ of $U$, we can find between the endpoints in $\partial \mathbf{D}$ of $\phi^{-1}(Q)$, in either circular direction, an element of $\mathscr{D}$. Thus, the domains $U_{1}$ and $U_{2}$ in $U$ cut off by $Q$ each have $\partial U_{i} \supset J$. Thus, for each prime end $\eta \in \partial \mathbf{D}$, defined by a chain of such crosscuts, we have $I(\eta)=J$.

$(4) \Rightarrow(5)$. Since all prime ends of $U$ have $I(\eta)=J$, there is no prime end of the first kind. It follows from Theorem 1.3 that the set of prime ends of the third kind and, consequently, the set of simple dense canals, is residual in $\partial \mathbf{D}$.

$(5) \Rightarrow(6)$. Trivial.

(6) $\Rightarrow(2)$. By definition, a simple dense canal is a prime end $\eta$ for which $P(\eta)=I(\eta)=J$. (Alternately, by [Ru, Theorem 3] the existence of a simple dense canal directly implies (1) $J$ indecomposable.)

3.5. Corollary. Suppose $f_{c}(z)=z^{2}+c$ with $J\left(f_{c}\right)$ an indecomposable continuum. Then no composant of $J\left(f_{c}\right)$ can contain the principal set of more than one prime end of the domain $U=\widehat{\mathbf{C}}-K\left(f_{c}\right)$.

Proof. The proof is similar to the proof of Proposition 11 in [R1], using the dense set $\mathscr{D}$ of Theorem 3.4(3). (Also see [Br, Theorem 3.3] for the general principle.)

3.6. Question. Is there a (quadratic) polynomial whose Julia set is an indecomposable continuum?

3.7. Question. Does Theorem 3.4 hold for all polynomials $f$ of degree $d \geq 2$ for which $J(f)$ is connected? (The problematic step is $(2) \Rightarrow(3)$.)

An affirmative answer to the following question would imply an affirmative answer to Question 3.7.

3.8. Question. Suppose $f$ is a polynomial of degree $d \geq 2$ with $J(f)$ connected. Let $U$ be the domain $\widehat{\mathbf{C}}-K(f)$. Suppose that $\phi: \widehat{\mathbf{C}}-\overline{\mathbf{D}} \rightarrow U$ is a uniformization such that $\phi(\infty)=\infty$ and $\phi$ conjugates $f$ to $h(z)=z^{d}$ on $\widehat{\mathbf{C}}-\overline{\mathbf{D}}$. Suppose further that for some prime end $\eta$ of $U, I(\eta)$ has interior in $J(f)$ (or, if you prefer, $I(\eta)=J(f))$. Does it follow that for each $\eta^{\prime} \in h^{-1}(\eta)$, $I\left(\eta^{\prime}\right)$ has interior in $J(f)$ ?

3.9. Remark. The second author [R3] has obtained theorems analogous to those in $\S 3$ for boundaries of Siegel disks.

\section{REFERENCES}

[B] B. Bielefeld, ed., Conformal dynamics problem list, preprint \#1990/1, Institute for Mathematical Sciences, SUNY-Stony Brook.

[Br] B. L. Brechner, On stable homeomorphisms and imbeddings of the pseudo arc, Illinois J. Math. 22 (1978), 630-661.

[BGM] B. L. Brechner, M. D. Guay, and J. C. Mayer, Rotational dynamics on cofrontiers, Continuum Theory and Dynamical Systems (M. Brown, ed.), Contemp. Math., vol. 117, Amer. Math. Soc., Providence, RI, 1991, pp. 39-48.

[CL] E. F. Collingwood and A. J. Lohwater, Theory of Cluster sets, Cambridge Tracts in Math. and Math. Physics, vol. 56, Cambridge Univ. Press, Cambridge, 1966. 
[DH1] A. Douady and J. H. Hubbard, Étude dynamique des polynômes complexes (première partie), Publ. Math. Orsay 2 (1984), 1-75.

[DH2] _ Étude dynamique des polynômes complexes (deuxième partie), Publ. Math. Orsay 4 (1985), 1-154.

[H] C. L. Hagopian, A fixed point theorem for plane continua, Bull. Amer. Math. Soc. 77 (1971), 351-354.

[HY] J. Hocking and G. Young, Topology, Addison-Wesley, Reading, MA, 1961.

[K1] J. Krasinkiewicz, On the composants of indecomposable plane continua, Bull. Polon. Acad. Sci. 20 (1972), 935-940.

[K2] - On internal composants of indecomposable plane continua, Fund. Math. 84 (1974), 255-263.

[MO] J. C. Mayer and L. G. Oversteegen, Denjoy meets rotation on an indecomposable cofrontier , preprint.

[M] J. Milnor, Dynamics in one complex variable: introductory lectures, preprint \#1990/5, Institute for Mathematical Sciences, SUNY-Stony Brook.

[P] G. Piranian, The boundary of a simply connected domain, Bull. Amer. Math. Soc. 64 (1958), 45-55.

[R1] J. T. Rogers, Jr., Intrinsic rotations of simply connected regions and their boundaries, Complex Variable Theory Appl. (to appear).

[R2] - Indecomposable continua, prime ends, and Julia sets, preprint.

[R3] - Singularities in the boundaries of local Siegel disks, preprint.

[Ru] N.E. Rutt, Prime ends and indecomposability, Bull. Amer. Math. Soc. 41 (1935), 265-273.

Department of Mathematics, University of Alabama at Birmingham, Birmingham, AlABAMA 35294-2060

E-mail address: mayer@math.uab.edu

Department of Mathematics, Tulane University, New Orleans, Louisiana 70118

E-mail address: mt1damf@vm.tcs.tulane.edu 